\title{
Infant with chronic bilateral retroauricular tumefaction (2009: 3a)
}

(C) European Society of Radiology 2008

\section{Case report}

A 3-year-old boy with a history of bilateral retroauricular tumefaction for the past 3 months and treated with several courses of anti-inflammatory agents and antibiotics was brought to the emergency ward.

A CT (Fig. 1) and MR-study (Fig. 2) were performed.

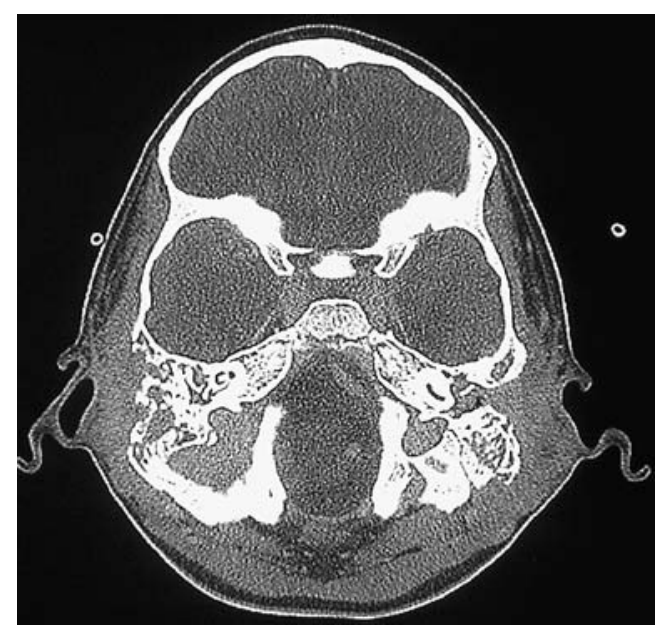

Fig. 1 CT image

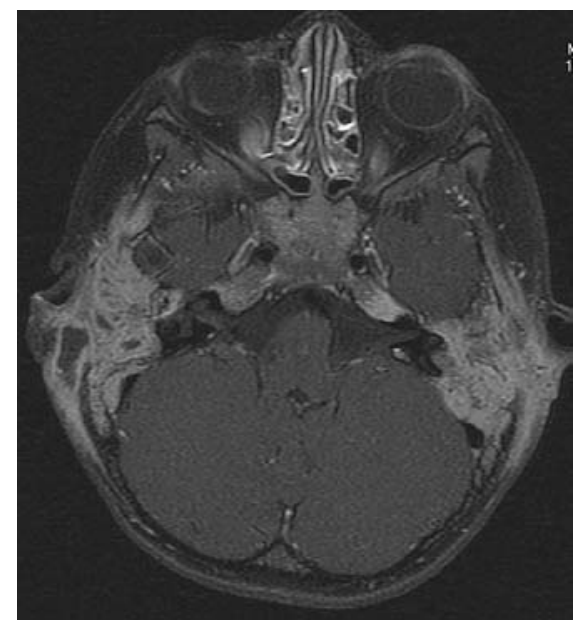

Fig. 2 Gadolinium-enhanced T1-weighted image

\section{What is the diagnosis?}

Readers are invited to supply one possible diagnosis via electronic means to: robert.hermans@uzleuven.be

The subject of the e-mail should include 'Interpretation Corner' and the number given above (2009: 3a).

You should include your name, title, address, fax and phone number.

Deadline: one clear calendar month from the distribution date.

Three months after the initial publication of the case history, the authors will publish the final diagnosis and a brief summary. The summary will describe exactly how the case was investigated at the host institution, how the diagnosis was established and the teaching points of the case in question.

The names of the first 25 radiologists submitting the correct diagnosis will be published (only one from any individual centre and none from the host institution!). 